\title{
Respiratory impedance in healthy subjects: baseline values and bronchodilator response
}

\author{
Ellie Oostveen ${ }^{1}$, Krisztina Boda², Chris P.M. van der Grinten ${ }^{3}$, Alan L. James ${ }^{4}$, \\ Sally Young ${ }^{4}$, Hans Nieland ${ }^{5}$ and Zoltán Hantos ${ }^{2}$
}

Affiliations: 'Dept of Respiratory Medicine, Antwerp University Hospital and University of Antwerp, Antwerp, Belgium. ${ }^{2}$ Dept of Medical Physics and Informatics, University of Szeged, Szeged, Hungary. ${ }^{3}$ Dept of Respiratory Medicine, Maastricht University Medical Centre, Maastricht, and ${ }^{5}$ Dept of Lung Function, Medisch Spectrum Twente, Enschede, The Netherlands. ${ }^{4}$ Dept of Pulmonary Physiology, Sir Charles Gairdner Hospital, Nedlands, Australia.

Correspondence: E. Oostveen, Antwerp University Hospital, Wilrijkstraat 10, Edegem-Antwerp, B-2650, Belgium. E-mail: ellie.oostveenduza.be

ABSTRACT Because of the minimal demand for cooperation by the subject, the forced oscillation technique is increasingly employed in routine lung function testing. However, comprehensive and deviceindependent values of respiratory impedance at baseline and after bronchodilation have not been established for healthy adults.

The aim of this multicentre study was to collect impedance data from 4 to $26 \mathrm{~Hz}$ in healthy Caucasian subjects between 18 and 80 years of age. Five different devices were employed to assess baseline values and the bronchodilator response.

Altogether, 368 subjects were examined. Despite adjustment for anthropometry, the impedance spectra differed in frequency dependence between the centres, and hence could not be pooled. However, resistance at all frequencies except 20 and $25 \mathrm{~Hz}$, and the low-frequency $(\leqslant 14 \mathrm{~Hz}$ ) values of reactance did not exhibit a centre dependence. The regression equations for resistance reflected a greater height dependence in males and a greater weight dependence in both males and females than those published previously. Bronchodilation resulted in a statistically significant decrease (11\%) in resistance and a 95th percentile equal to a $32 \%$ decrease in resistance at low frequency.

We conclude that rigorous calibration procedures should be developed to ensure data compatibility. Furthermore, new reference equations based on different setups are recommended to replace those established with a single device.

@ERSpublications

New prediction equations for respiratory impedance in adults have been developed based on multiple devices http://ow.ly/p9t4e

For editorial comments see page 1435.

This article has supplementary material available from www.erj.ersjournals.com

Received: Aug 102012 | Accepted after revision: Feb 062013 | First published online: April 182013

Conflict of interest: None declared.

Copyright @ERS 2013 


\section{Introduction}

Measurement of the mechanical properties of the respiratory system using the forced oscillation technique (FOT) is increasingly employed in lung function laboratories. The main advantage of the FOT is ease of application: the oscillatory signal is superimposed on spontaneous breathing and, hence, no special breathing manoeuvres are required.

This minimal demand for cooperation has made the FOT especially attractive in paediatric lung function testing, where a number of studies have been performed to establish normative data using various FOT devices $[1,2]$, the results proving largely coherent. In contrast, there have been only a few reports on normative respiratory impedance $(Z \mathrm{rs})$ data in healthy adults, and some involved only relatively young or only elderly subjects [3,4], or only a small number of oscillation frequencies were investigated [5]. Moreover, the criteria for selection of the subjects were not always reported or the sample population was limited to a rather narrow specific subgroup of subjects $[3,6]$. Equally important, most such normative studies were conducted in the same laboratory, using the same forced oscillation setup, the Oscillaire, developed by LÀNDSÉR and co-workers [3,6-8], which is no longer available.

Although international guidelines have been developed concerning use of the FOT in clinical practice [1], appropriate reference data derived from a healthy population must be available prior to clinical use, preferably obtained by using equipment and measurement procedures similar to those employed in the clinical setting. Additionally, data on the bronchodilator response in healthy subjects should be collected for the appropriate evaluation of reversibility tests in patients.

The goal of the present study was therefore to collect baseline values of $\mathrm{Zrs}$ and the bronchodilator response with different FOT devices, and to develop reference ranges of respiratory resistance and reactance as a function of frequency $(f)$ for healthy subjects aged 18-80 years. With this aim, three commercial FOT devices and two custom-made FOT setups were used in this five-centre (C1-C5) study for the measurement of $Z$ rs.

\section{Methods}

Subjects

The healthy, nonsmoking Caucasian adults ( $\geqslant 18$ years of age) included in the study had no history of pulmonary or cardiac disease, and no current wheezing, breathlessness, cough, phlegm production, hyperresponsive airways or recent respiratory tract infection. Ex-smokers with a smoking history of $>10$ pack-years were excluded. Each centre was requested to include at least five subjects of each sex per decade of age, with a total number of $\geqslant 60$ subjects per centre. Except for Perth (Australia), where the invited subjects had previously taken part in the Busselton Health Studies [9], all the centres recruited subjects from among the local hospital or university staff and their family members. All subjects gave their written informed consent and the protocol was approved by the local ethical committees.

\section{Measurements}

Impedance measurements

Zrs was measured using two home-made setups in Antwerp, Belgium (C1) and Szeged, Hungary (C3), while ROS Oscilink (SensorMedics, Bilthoven, the Netherlands), i2M (Chess mT, Ghent, Belgium) and IOS (Jaeger, Würzburg, Germany) devices were used in Enschede, the Netherlands (C2), Perth, Australia (C4) and Maastricht, The Netherlands (C5). The measurements were made in accordance with recent European Respiratory Society (ERS) guidelines [1]. The averages and standard deviations of three to five technically acceptable Zrs measurements from each subject were retained for further analysis. Specifications on the oscillatory signal type, frequency content and recording time of the different setups are summarised in table 1.

\section{TABLE 1 Characteristics of the different forced oscillation technique devices}

\begin{tabular}{lccccc} 
Centre & Setup & Signal type & Frequency range Hz & Frequency resolution Hz & Recording time s \\
\hline C1 & Custom-made & Pseudorandom & $4-32$ & 2 & 1 \\
C2 & ROS Oscilink & Pseudorandom & $4-30$ & 2 & 16 \\
C3 & Custom-made & Pseudorandom & $2-26$ & 2 & 20 \\
C4 & i $^{*}$ & Pseudorandom & $4-48$ & 5 & 8 \\
C5 & IOS $^{+}$ & Train of impulses & $5-35$ & 30 \\
\hline
\end{tabular}

C1: Antwerp, Belgium; C2: Enschede, the Netherlands; C3: Szeged, Hungary; C4: Perth, Australia; C5: Maastricht, the Netherlands. \#: SensorMedics, Bilthoven, the Netherlands; ": Chess mT, Ghent, Belgium; ${ }^{+}$: Jaeger, Würzburg, Germany. 


\section{Spirometry}

The subjects performed repeated spirometric manoeuvres that met the American Thoracic Society standards [10] in C4 or the ERS standards [11] in C1-C3 and C5.

Zrs data were collected prior to spirometry. The measurements of the baseline values of $Z$ rs and forced expiration were repeated after $15 \mathrm{~min}$ to assess short-term repeatability. Subsequently, $400 \mu \mathrm{g}$ of salbutamol was administered through a spacer and the lung function measurements were repeated 15 min thereafter.

\section{Reference impedance}

A high-resistance $\left(8.9 \mathrm{hPa} \cdot \mathrm{s} \cdot \mathrm{L}^{-1}\right)$ reference impedance was circulated among the centres to check the accuracy of the different FOT setups.

The different FOT devices and calibration methods, the spirometers, and the measurements of the reference impedance are described in the online supplementary material.

\section{Statistical methods}

Anthropometric data

The intercentre comparisons of anthropometric and spirometric data were performed with one-way ANOVA with Fisher's post hoc least significant difference analysis.

\section{Impedance data}

The intercentre comparisons of the Zrs spectra were made with repeated-measures ANOVA. Because of the skewed distributions, resistance $(R \mathrm{rs})$ and reactance $(X \mathrm{rs})$ of the respiratory system were analysed after logarithmic transformations: $\ln (R \mathrm{rs})$ and $\ln (4-X \mathrm{rs})$, respectively. To take into account the different sets of oscillation frequencies included in the different FOT setups and to model the covariance structures of the simultaneously measured data, mixed-model ANOVA [12] was employed. Prediction equations were derived from multiple linear regression models.

From the short-term variability of the lung function data, the repeatability measure defined as the withinsubject within-occasion variability was assessed in 302 subjects ( $82 \%$ of the total population; 150 male and 152 female). This variability was expressed as the coefficient of repeatability, defined as twice the standard deviation of the difference of the two baseline measurements made $15 \mathrm{~min}$ apart [2]. The coefficient of repeatability defines the limits within which $95 \%$ of the differences between two measurements will lie if the bias is zero. Coefficient of repeatability was used to estimate the number of responders to the bronchodilation administration. Since the largest changes in $\mathrm{Zrs}$ occur in asthmatic patients at low frequency [1], this part of the analysis was restricted to Rrs and Xrs at low frequency in order to compare with the change in forced expiratory volume in $1 \mathrm{~s}$ (FEV1). The 95th percentile of the bronchodilator response in our population was also calculated.

\section{Results}

A total of 368 subjects ( $88 \%$ lifetime nonsmokers) were included in the study; the ex-smokers had an average smoking history of 4.4 pack-years. The ex-smokers and never-smokers, corrected for anthropometry, were not different in terms of spirometry results and $Z_{\text {rs }}$ data. The anthropometric characteristics of the studied subjects are listed in table 2; the only significant intercentre differences were that the subjects from C2 were younger, taller and lighter, and the subjects from C4 were older than those from the other centres.

As expected from the anthropometric data, higher absolute spirometric values were observed in the subjects from C2, but they were not significantly different when the results were expressed relative to the predicted value (table 2). In contrast, the C4 subjects displayed significantly lower spirometric volumes than those from all other centres. The ratios of FEV1/vital capacity (VC) were slightly lower for the C5 subjects than for those from the other centres.

\section{Quality control of the FOT equipment}

The impedance of the reference device was assessed with the different FOT setups and proved to be within $5 \%$ and $5^{\circ}$ of the expected magnitude and phase shift, respectively, at all frequencies for all devices except that of $\mathrm{C} 4$, where a deviation of $>5 \%$ from the expected magnitude was observed at frequencies $\geqslant 10 \mathrm{~Hz}$ (online supplementary fig. S1).

\section{Centre dependence of Zrs data}

Mean values of Rrs and $X r$ s at the different centres after adjustment for the covariates height, age and weight are plotted in figure 1; some systematic intercentre differences are apparent in the frequency dependences of 
TABLE 2 Anthropometric characteristics and baseline lung function data for the subjects included at each centre

Centre

\begin{tabular}{|c|c|c|c|c|c|}
\hline & $\mathrm{C} 1$ & $\mathrm{C} 2$ & C3 & C4 & $\mathrm{C5}$ \\
\hline Subjects n (\% male) & $66(52)$ & $61(46)$ & $71(49)$ & $108(50)$ & 62 (47) \\
\hline Age years & $47.9 \pm 17.3$ & $42.6 \pm 15.2^{*}$ & $49.1 \pm 17.4$ & $54.8 \pm 15.7^{*}$ & $49.5 \pm 17.3$ \\
\hline Height $\mathrm{cm}$ & $171.8 \pm 9.9$ & $174.7 \pm 9.0^{*}$ & $170.3 \pm 10.8$ & $170.5 \pm 9.8$ & $170.8 \pm 9.2$ \\
\hline BMI $\mathrm{kg} \cdot \mathrm{m}^{-2}$ & $26.3 \pm 4.2$ & $24.0 \pm 3.1^{*}$ & $25.4 \pm 4.2$ & $26.5 \pm 4.6$ & $25.5 \pm 3.6$ \\
\hline FEV1 L & $3.6 \pm 1.0$ & $3.8 \pm 0.9^{\#}$ & $3.4 \pm 0.9$ & $3.1 \pm 0.9^{*}$ & $3.4 \pm 0.9$ \\
\hline FEV $1 \%$ pred & $105 \pm 13$ & $102 \pm 12$ & $102 \pm 12$ & $95 \pm 13^{* * *}$ & $104 \pm 13$ \\
\hline FEV1 z-score & $0.39 \pm 0.95$ & $0.15 \pm 0.93$ & $0.17 \pm 0.90$ & $-0.33 \pm 0.88 * * *$ & $0.30 \pm 0.93$ \\
\hline VC L & $4.4 \pm 1.2$ & $4.7 \pm 1.1^{\#}$ & $4.2 \pm 1.2$ & $3.9 \pm 1.1^{*}$ & $4.4 \pm 1.1$ \\
\hline VC \% pred & $104 \pm 12$ & $103 \pm 10$ & $101 \pm 12$ & $95 \pm 12^{* * *}$ & $107 \pm 12^{\#}$ \\
\hline VC z-score & $0.25 \pm 0.87$ & $0.19 \pm 0.77$ & $0.04 \pm 0.88$ & $-0.36 \pm 0.82^{* * *}$ & $0.43 \pm 0.83^{\#}$ \\
\hline FEV1/VC \% & $81 \pm 5$ & $80 \pm 7$ & $82 \pm 6$ & $79 \pm 6$ & $78 \pm 6^{+}$ \\
\hline FEV1/VC z-score & $0.16 \pm 0.72$ & $-0.08 \pm 1.07$ & $0.17 \pm 0.83$ & $0.00 \pm 0.78$ & $-0.28 \pm 0.74^{\mathrm{s}}$ \\
\hline
\end{tabular}

Data are presented as mean \pm SD, unless otherwise stated. The Global Lungs Initiative reference equations [13] were used to express the spirometry data as percentages of those predicted (\% pred) and as z-scores. C1: Antwerp, Belgium; C2: Enschede, the Netherlands; C3: Szeged, Hungary; C4: Perth, Australia; C5: Maastricht, the Netherlands; BMI: body mass index; FEV1: forced expiratory volume in 1 s; VC: vital capacity. ${ }^{*}: \mathrm{p}<0.05{ }^{* * *}: \mathrm{p}<0.001$ versus all the other centres; ${ }^{*}: \mathrm{p}<0.01$ versus the data from $\mathrm{C} 3 ;{ }^{\top}: \mathrm{p}<0.05$ versus the data from $\mathrm{C} 1$ and $\mathrm{C} 3$; ${ }^{+}: \mathrm{p}<0.05$ versus the data from $\mathrm{C} 1, \mathrm{C} 2$ and $\mathrm{C} 3 ;{ }^{\S}: \mathrm{p}<0.05$ versus the data from $\mathrm{C} 1, \mathrm{C} 3$ and $\mathrm{C} 4$.

Rrs and Xrs. As described in detail in the online supplementary material, mixed-model analysis was applied to choose the autoregressive covariance structure with which to model the correlation between spectra. Mixed models with the interaction centre $\times$ frequency and the covariate combinations height-age-weight and height-age-body mass index (BMI) were addressed. Since the latter combination did not improve the model performance, height, age and weight were retained for the subsequent analysis. The dependences of the $R \mathrm{rs}$ and the $X \mathrm{rs}$ data on sex, frequency and centre were analysed on $\ln (R \mathrm{rs})$ and $\ln (4-X \mathrm{rs})$. Linear interpolation of $Z$ rs values between some frequency values was necessary to facilitate the combination of data from the different centres. The frequency dependence of $Z$ rs was significantly different in the various centres, thus the full set of $Z$ rs spectra from all the centres could not be combined; however, data pooling was possible for Rrs (with the exceptions of 20 and $25 \mathrm{~Hz}$ in the females and $25 \mathrm{~Hz}$ in the males) and for Xrs up to $14 \mathrm{~Hz}$ in both sexes. Exclusion of the data from C5 eliminated the significant differences in Rrs, but not in Xrs. The C5 data were retained for further analysis.

\section{Prediction equations}

Values of single-frequency Rrs and Xrs, the resonant frequency (fres), the reactance curve area (Ax) [14] and the mean Rrs (Rmean) were included in the predictions on height, age and weight. The coefficients of the prediction equations and the residual standard deviations for $\ln (R \mathrm{rs})$ and $\ln (4-X \mathrm{rs})$ are listed in tables 3 and 4 , respectively. Although age did not prove to be a significant contributor to the Rrs values at high frequency $(>12 \mathrm{~Hz})$ for the females, it was not omitted from the model for reasons of consistency of the predicted Rrs as a function of frequency. However, age did not contribute significantly to the prediction of the Xrs values for the males at any frequency, and was therefore omitted from this particular prediction. Unlike the frequency dependence of $R \mathrm{rs}$, the Rmean values were not statistically different between the centres, and the $A \mathrm{X}$ calculated either between $4 \mathrm{~Hz}$ and $\mathrm{fres}_{\mathrm{AX} 4)}$ or between $5 \mathrm{~Hz}$ and fres $(A \times 5)$ were also centreindependent. Although there was a centre dependence in fres, the differences between the centres were small (the largest difference was $<2 \mathrm{~Hz}$ ) and considered clinically irrelevant. Calculation examples for the predicted median values and upper/lower limits of the normal values are given in the online supplementary material.

\section{Baseline variability of Zrs}

The variability in $R \mathrm{rs}$, expressed in both absolute and relative terms, did not differ between the centres or between the sexes. Furthermore, no systematic differences were found between the two baseline measurements in Rrs and in Xrs. The coefficient of repeatability values of Rrs at low frequency $(n=302)$ and that of FEV1 $(n=179)$ are reported in table 5. Bland-Altman analysis [15] revealed that the absolute difference between the repeated baseline measurements was related to the magnitude of the baseline value of Rrs. The relative difference between the two baseline measurements, however, was independent of the magnitude of Rrs (fig. 2). 

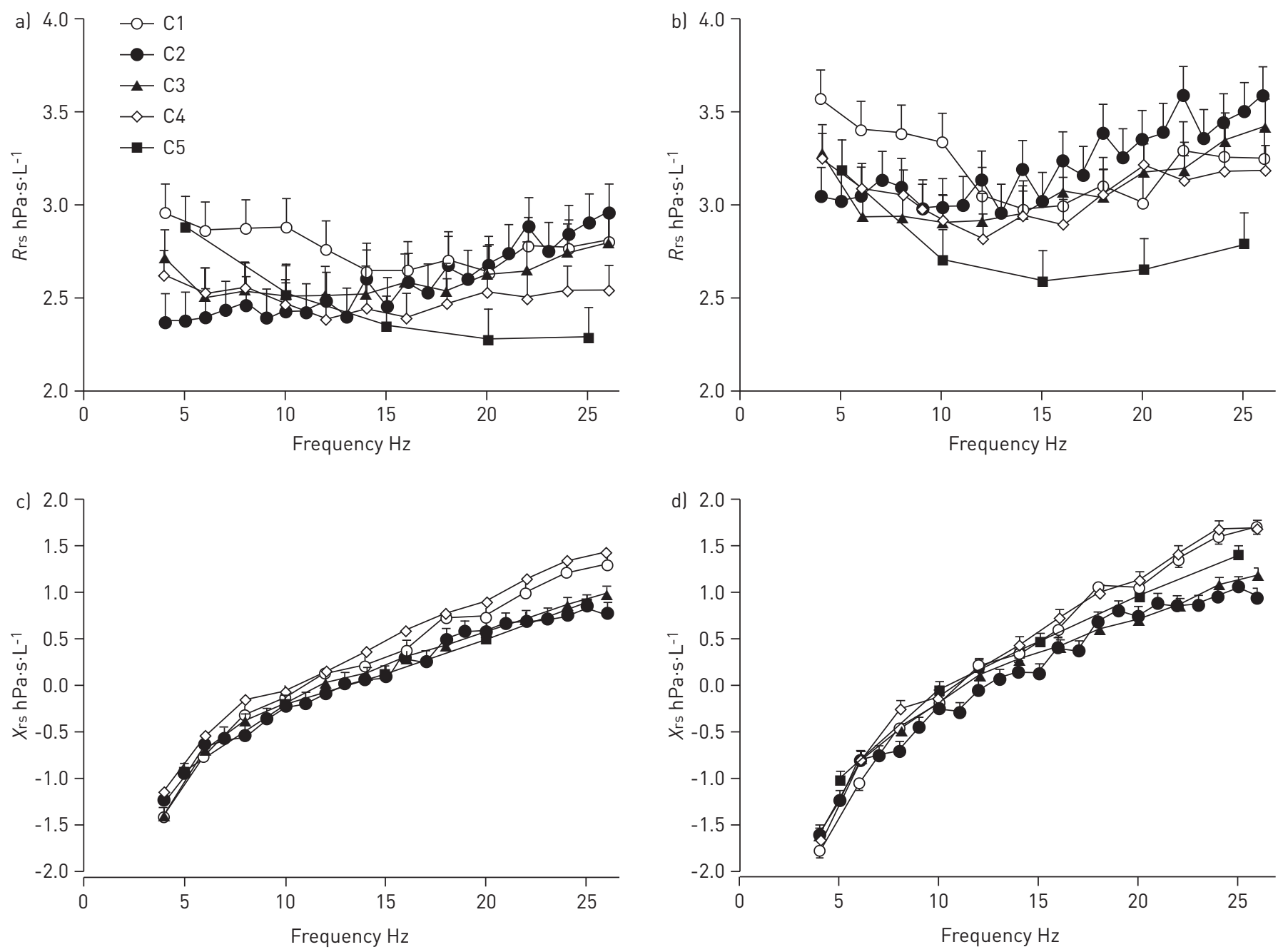

FIGURE 1 Mean \pm SEM values of respiratory a, b) resistance (Rrs) and c, d) reactance (Xrs) obtained from healthy adult males $(\mathrm{a}, \mathrm{c})$ and females $(\mathrm{b}, \mathrm{d})$ through the use of different forced oscillation technique devices at five different centres (C1: Antwerp, Belgium; C2: Enschede, the Netherlands; C3: Szeged, Hungary; C4: Perth, Australia; C5: Maastricht, the Netherlands), adjusted for height, age and weight in the mixed-model analysis.

\section{Bronchodilator response}

There was no centre dependence of the bronchodilator effect as assessed with FOT. Bronchodilation induced a significant decrease in $\operatorname{Rrs}$ at all frequencies (mean decrease $11 \%$ ) and an increase in $X \mathrm{rs}$ at 4, 5 and $6 \mathrm{~Hz}$ and FEV1 ( $\mathrm{p}<0.001$, mixed-model ANOVA), whereas vital capacity did not change. The changes in $R$ rs were more pronounced than those in $X_{\text {rs }}$ (fig. 3).

The relative coefficient of repeatability was used to identify responders to bronchodilation. Rrs at $5 \mathrm{~Hz}$ identified $36 \%$ of the healthy subjects as positive responders to salbutamol and $3 \%$ of the subjects as contraresponders. By contrast, FEV1 identified $17 \%$ of the studied subjects as positive responders and $1 \%$ as contraresponders (online supplementary table S1). Only $28(8 \%)$ subjects were positive responders according to both methods. Xrs at low frequency was markedly less sensitive for detection of the bronchodilator response compared to Rrs at low frequency (online supplementary table S1).

In order to help define a positive response in asthmatic patients, the 95th percentile of the bronchodilator response in healthy adults was established. The 95th percentiles for the absolute and relative changes in Rrs and $X \mathrm{rs}$ at low frequencies due to bronchodilation are reported in table 5. For example, the 95th percentile response in $\mathrm{Rrs}$ at $4 \mathrm{~Hz}$ was $1.41 \mathrm{hPa} \cdot \mathrm{s} \cdot \mathrm{L}^{-1}$, corresponding to a relative decrease of $33 \%$ (table 5).

\section{Discussion}

As far as we are aware, this is the first study in which baseline and post-bronchodilator Zrs data were collected from healthy adult subjects at multiple centres. The Rmean values found in our study are close to the published data $[3,4,6,8]$. In accordance with earlier findings, females displayed larger values of $\operatorname{Rrs}$ than 
TABLE 3 Prediction equations for respiratory resistance ( $R \mathrm{rs}$ ) at the different frequencies ( $f$ ), and the average resistance (Rmean) for males and females

\begin{tabular}{|c|c|c|c|c|c|c|c|c|c|c|}
\hline$f \mathrm{~Hz}$ & \multicolumn{5}{|c|}{ Males } & \multicolumn{5}{|c|}{ Females } \\
\hline 5 & 5.327 & -3.032 & -0.00381 & 0.01390 & 0.2803 & 2.591 & -1.461 & 0.00279 & 0.01221 & 0.2657 \\
\hline 6 & 5.454 & -3.079 & -0.00482 & 0.01366 & 0.2662 & 2.556 & -1.464 & 0.00310 & 0.01222 & 0.2648 \\
\hline 8 & 5.256 & -2.972 & -0.00418 & 0.01352 & 0.2669 & 2.642 & -1.488 & 0.00351 & 0.01119 & 0.2681 \\
\hline 14 & 4.402 & -2.393 & -0.00382 & 0.01091 & 0.2618 & 2.338 & -1.213 & 0.00266 & 0.00936 & 0.2528 \\
\hline 15 & 4.181 & -2.286 & -0.00328 & 0.01066 & 0.2649 & 2.426 & -1.245 & 0.00144 & 0.00927 & 0.2562 \\
\hline 16 & 4.373 & -2.337 & -0.00429 & 0.01033 & 0.2609 & 2.423 & -1.198 & 0.00169 & 0.00861 & 0.2486 \\
\hline 18 & 3.961 & -2.052 & -0.00422 & 0.00937 & 0.2609 & 2.203 & -1.027 & 0.00199 & 0.00798 & 0.2522 \\
\hline $20^{\#}$ & 3.540 & -1.824 & -0.00330 & 0.00888 & 0.2638 & 2.482 & -1.122 & 0.00135 & 0.00695 & 0.2467 \\
\hline 22 & 3.768 & -1.834 & -0.00457 & 0.00771 & 0.2561 & 2.259 & -0.951 & 0.00086 & 0.00682 & 0.2501 \\
\hline
\end{tabular}

$\ln (\operatorname{Rrs}(f))=\mathrm{a}+\mathrm{b} \times$ height $+\mathrm{c} \times$ age $+\mathrm{d} \times$ weight. Units: $\operatorname{Rrs}: \mathrm{hPa} \cdot \mathrm{s} \cdot \mathrm{L}^{-1}$; height: $\mathrm{m}$; age: years; weight: $\mathrm{kg}$. Males: age range: $18-84$ years; height range: 1.59-1.97 m; weight range: 54-128 kg. Females: age range: 19-81 years; height range: 1.47-1.88 m; weight range: 43-111 kg. The residual standard deviation (RSD) was calculated from the regression of transformed data. \# : data on females from C5 omitted.

those of males. The impedance data of our healthy adult subjects were described by variables similar to those used previously in prediction equations: sex, height, age and weight.

\section{Study population}

The age and sex distributions of the population were well controlled; males and females contributed virtually equally, and the participants of each sex were evenly distributed between 18 and 80 years of age. All subjects included participated in spirometry. The FEV1 and VC values of the subject population were close to the expected values (table 2). The baseline spirometry data from the individual centres were essentially similar, with one exception: the $\mathrm{C} 4$ subjects furnished values which were significantly lower than those at the other centres, but still within the expected range [13].

Obesity was not an exclusion criterion in our study. The average BMI was 25.3 and $26.1 \mathrm{~kg} \cdot \mathrm{m}^{-2}$ for females and males, respectively; values close to those reported by the World Health Organization for adults [16]. Rrs

\section{TABLE 4 Prediction equations for reactance $(X r s)$ data at the different frequencies $(f)$ for males and females}

\begin{tabular}{|c|c|c|c|c|c|c|c|c|c|c|}
\hline$f \mathrm{~Hz}$ & \multicolumn{5}{|c|}{ Males } & \multicolumn{5}{|c|}{ Females } \\
\hline 5 & 2.683 & -0.703 & 0 & 0.00190 & 0.0728 & 2.373 & -0.607 & 0.00150 & 0.00312 & 0.0814 \\
\hline 6 & 2.407 & -0.606 & 0 & 0.00234 & 0.0701 & 2.212 & -0.577 & 0.00144 & 0.00373 & 0.0806 \\
\hline 8 & 2.180 & -0.497 & 0 & 0.00187 & 0.0654 & 1.916 & -0.396 & 0.00074 & 0.00293 & 0.0848 \\
\hline 14 & 2.265 & -0.679 & 0 & 0.00310 & 0.0891 & 1.500 & -0.351 & 0.00228 & 0.00405 & 0.1088 \\
\hline $\ln$ (fres) & 5.070 & -1.904 & 0 & 0.00864 & 0.2498 & 3.415 & -1.104 & 0.00354 & 0.01001 & 0.2549 \\
\hline $\ln (A \times 4)$ & 9.034 & -5.288 & 0 & 0.01719 & 0.5670 & 5.778 & -3.785 & 0.00960 & 0.02220 & 0.5673 \\
\hline $\ln (A \times 5)$ & 9.730 & -6.107 & 0 & 0.02122 & 0.7266 & 5.490 & -4.122 & 0.00960 & 0.02836 & 0.6942 \\
\hline
\end{tabular}

$\ln \left(4-X_{r s}(f)\right)=a+b \times$ height $+c \times$ age $+\mathrm{d} \times$ weight. Units: $X r s: ~ h P a \cdot s \cdot L^{-1}$; height: $\mathrm{m}$; age: years; weight: $\mathrm{kg}$. Males: age range: 18-84 years; height range: 1.59-1.97 m; weight range: 54-128 kg. Females: age range: 19-81 years; height range: 1.47-1.88 m; weight range: 43-111 kg. fres: resonant frequency; $A \times 4$ and $A \times 5$ : area under the reactance curve from 4 and $5 \mathrm{~Hz}$, respectively. fres, $A \times 4$ and $A \times 5$ data were logarithmically transformed to take into account the skewness of the data. The residual standard deviation (RSD) was calculated from the regression of transformed data. 
TABLE 5 The short-term repeatability and the bronchodilator response for respiratory resistance and reactance at low frequency and forced expiratory volume in $1 \mathrm{~s}$ (FEV1), as observed in 368 healthy adult subjects

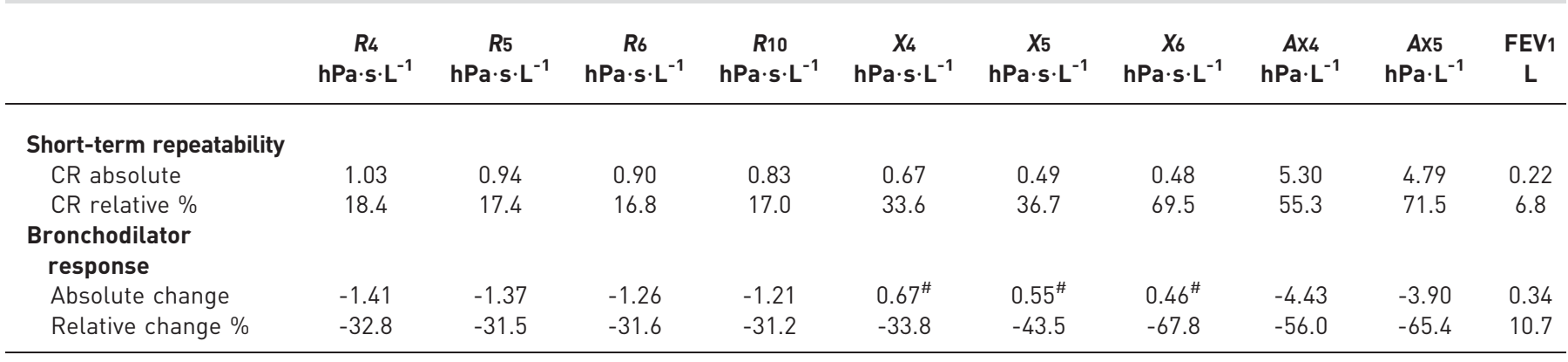

The coefficients of repeatability (CR) and 95th percentile of the change in resistance and reactance at low frequency and FEV 1 are expressed in absolute and relative terms. For the bronchodilator response, the absolute change was expressed as post-bronchodilator value minus prebronchodilator value and the relative change as absolute change/pre-bronchodilator value. $R 4, R 5, R 6$ and $R 10$ : respiratory resistance at $4,5,6$ and $10 \mathrm{~Hz}$, respectively; $X_{4}, X_{5}$ and $X_{6}$ : respiratory reactance at 4,5 and $6 \mathrm{~Hz}$, respectively; $A X_{4}$ and $A x_{5}$ : area under the reactance curve calculated from 4 and $5 \mathrm{~Hz}$, respectively. " : the fifth percentile was determined.

is expected to rise and $\mathrm{Xrs}$ to fall with the level of obesity $[17,18]$, but BMI did not seem to be a stronger predictor than weight in our study.

\section{Comparison with earlier predictions}

Our predicted values for $R$ mean are compared with previous predictions in figure 4 . The current equations predict higher values of $R$ mean than most of the earlier predictions [4, 5, 6, 8, 19] for short and young males, and lower values for tall males, the predicted Rmean therefore exhibiting a larger height dependence, whereas lower values of $R$ mean for young females were found, but with a similar height dependence, compared to most previously published equations.

A comparison of the present and previous predictions for Rrs and $X \mathrm{rs}$ at individual frequencies is difficult, since in most of the literature reports $Z$ rs data were described by polynomials of frequency $[3,6,8,20]$ or linear regression determining the frequency dependence of Rrs and its extrapolated zero-frequency value [21]. A similar comparison of $R \mathrm{rs}$ as a function of weight with earlier predictions is presented in online supplementary figure S6.

The present study allows the prediction of Rrs between 4 and $26 \mathrm{~Hz}$ and $\mathrm{Xrs}$ at $\leqslant 14 \mathrm{~Hz}$ on the basis of data pooled from the five centres. Zrs data, and especially those measured at low frequency, are sensitive to bronchial obstruction and constriction [1], and the newly derived prediction equations adequately cover these frequency ranges. Measured data can be considered abnormal if the values of $R \mathrm{rs}, A \mathrm{x}$ or fres is greater or $X_{\mathrm{rs}}$ is lower than the corresponding $95 \%$ confidence limit, i.e. the reference value $\pm 1.64 \times$ residual standard deviation (tables 3 and 4 , and the online supplementary material).

FIGURE 2 Bland-Altman plot [15] of the repeatability of two baseline measurements of the resistance at $5 \mathrm{~Hz}$ (R5), made 15 min apart. Data are plotted as the relative difference between the two measurements versus the mean value of the two measurements. The solid line indicates the bias and the dashed lines indicate the upper and lower limits of agreement.

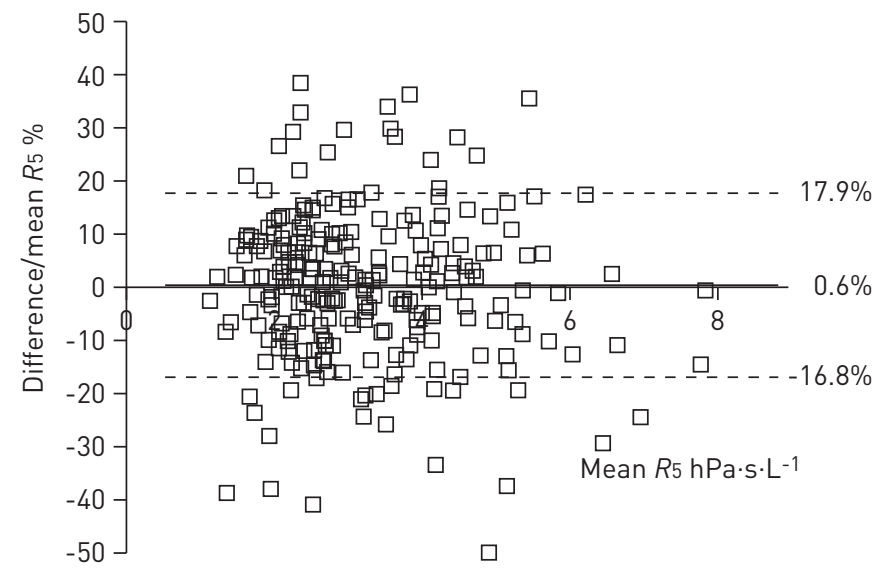




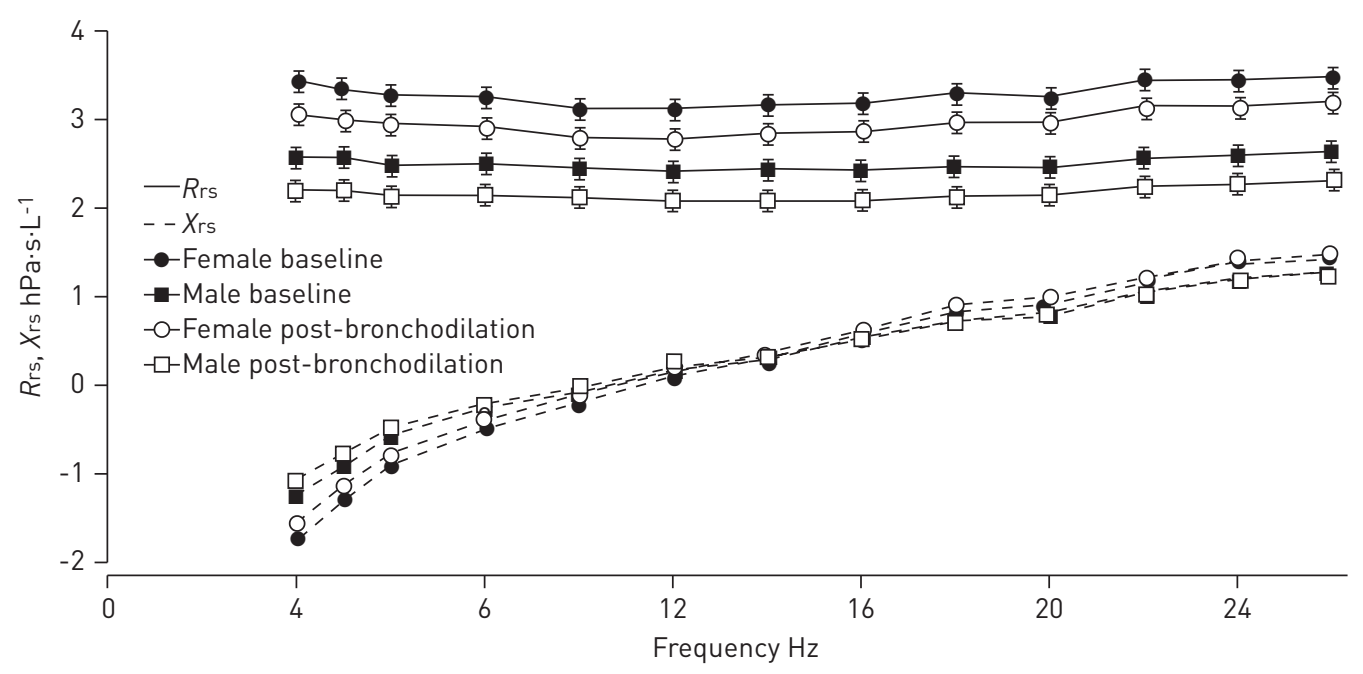

FIGURE 3 Mean \pm SE values of the respiratory resistance $(R \mathrm{rs})$ and reactance $(X \mathrm{rs})$ in healthy females $(\mathrm{n}=188)$ and males $(\mathrm{n}=180)$ at baseline and after bronchodilation.

\section{Equipment dependence}

Whereas all manufacturers stress the uniqueness of their devices, their users expect all FOT setups to comply with the ERS guidelines [1] and that the results obtained with the various setups should be comparable. Thus, it was an underlying assumption of the current study that the $Z_{\text {rs }}$ data measured in the different centres could be pooled. To test this assumption, a reference impedance was first sent to each of the centres in order to test the accuracy of the equipment. While all setups recovered the reference value more or less accurately, it should be noted that routine calibration devices usually have much lower impedance values $\left(\sim 2 \mathrm{hPa} \cdot \mathrm{s} \cdot \mathrm{L}^{-1}\right)$, which allow only a less rigorous testing.

The possibility of a device dependence in the $Z$ rs data was tested next. The statistical analysis demonstrated that the frequency dependence of the Rrs data on these healthy subjects differed in the various centres (fig. 1). However, despite anthropometry adjustment, there still might have been differences between the studied populations. This possible effect cannot be distinguished from the impact of device differences. The device at C5 was found to provide outlying Rrs data at 20 and $25 \mathrm{~Hz}$, and their Rrs data therefore exhibited a more marked negative frequency dependence than those from the other centres. Omission of C5 data from the regression changed the coefficients in the prediction equations of Rrs to a negligible extent (data not shown). However, it is important to point out that the interpretation of the negative frequency dependence of the Rrs data in terms of the function of the small airways $(R 5-R 20)$ and that of the large airways $(R 20)$ has been associated in the literature with this particular device $[22,23]$. The present finding, that the frequency dependence of Rrs is device-dependent, seriously challenges this simplistic interpretation.

The Xrs data at frequencies above fres were also centre dependent, although there was no single outlying centre or device; the centres rather differed pairwise (online supplementary fig. S1). The most probable explanation for this deviating $X \mathrm{rs}$ at high frequency is a difference in the inertance of the connecting tubes/ mouthpiece. These differences in construction and the correction procedures, which are not always transparent for the users, have less influence on the Xrs data at low frequencies.

A too-low common mode rejection ratio of the flowmeter may be an important determinant of a distorted measurement of the flow at high frequency [24]. Unfortunately, the common mode rejection ratio of the commercial devices was not specified and, in most cases, could not easily be checked by the users. However, all setups accurately measured the high reference resistance at all frequencies (online supplementary fig S1). Nevertheless, the frequency dependence of Rrs of healthy subjects after adjustment for anthropometry was shown to be centre, and thus device, dependent (fig. 1). This discrepancy might then result from differences in instrumentation and signal/data processing procedures, whose details are unavailable for the commercial devices. Perhaps most importantly, we implemented a calibration check procedure without pressure and flow fluctuations similar to those from spontaneous breathing, and hence the possibly different nonlinear performances of the FOT setups were not tested.

Previous studies have suggested that the frequency dependence of Rrs is a markedly sensitive index that permits differentiation between health and disease and an assessment of the disease severity [7, 25-27]. 

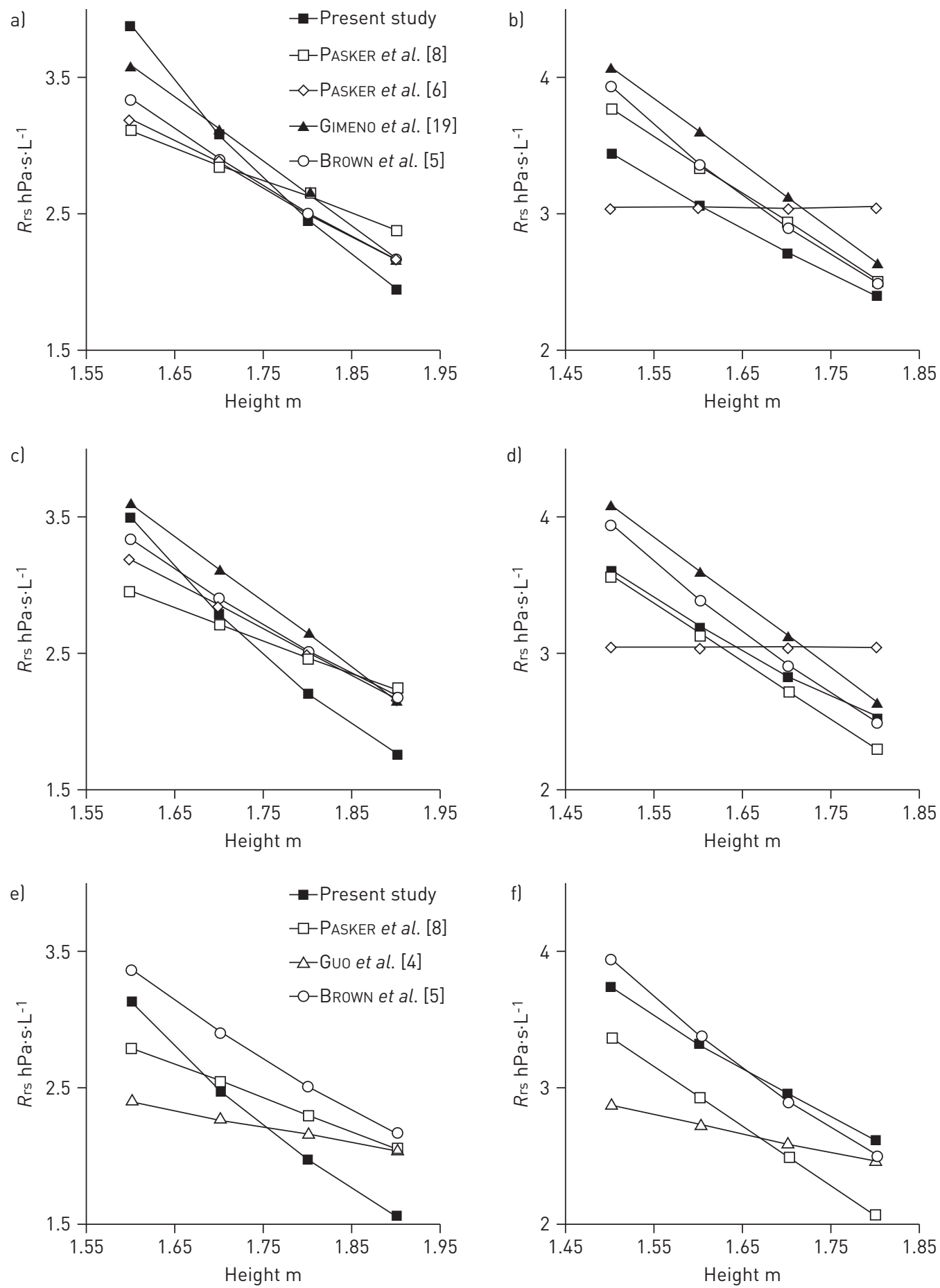

FIGURE 4 Comparison of the predicted mean values of respiratory resistance (Rrs) (Rmean) for males and females from the present study and those from previously published studies $[4,5,6,8,19]$, as functions of height at different ages. a) male, 20 years; b) female, 20 years; c) male, 50 years; d) female, 50 years; e) male, 80 years; f) female, 80 years. Weight was fixed at 80 and $65 \mathrm{~kg}$ in males and females, respectively. Predictions for Rrs at $19 \mathrm{~Hz}$ were computed from Brown et al. [5], since Rrs at $20 \mathrm{~Hz}$ was closest to Rmean in our dataset.

However, as noted above, our results indicate that this index is device dependent. We therefore suggest that the optimum way to describe abnormalities in $Z_{\text {rs }}$ is to look for an increase in Rrs and a decrease in $X_{r s}$ at low frequency only. Ax and fres are additional indices via which to characterise abnormal Xrs. 


\section{Baseline variability and bronchodilator response}

Use of the coefficients of repeatability as threshold values to define a positive (or negative) response to bronchodilation implies that, by definition, $5 \%$ of the studied subjects are expected to be marked as positive or negative responders if the intervention has no effect. The number of positive responders was much larger than $5 \%$, indicating that the bronchomotor tone changed in a significant proportion of our healthy adults. $R$ rs at low frequency detected twice as many positive responders than FEV1 did (36\% versus 17\%). The number of contra-responders with either index was $<5 \%$, but Rrs detected more contra-responders than FEV1 did (online supplementary table S1). Being more sensitive than FEV1 for detection of a change in bronchomotor tone in the clinical setting, Rrs has been proposed for broncho-challenge testing, but not for reversibility testing [1].

Perhaps the most important result of the bronchodilation part of our study is the 95th percentile of the response in our group of healthy adult subjects, which was equal to $\sim 32 \%$ for $R$ rs at low frequency. The bronchodilator response of healthy adults is largely unknown. We are aware of only one study in which subjects screened in an allergy and asthma clinic were assigned to a healthy control group [23]. They exhibited an average decrease of $15 \%$ in $R 5$ after bronchodilation, a value close to the decrease of $11 \%$ in the present study. The 95th percentile of the response in our group of healthy adults is slightly lower than the values previously reported for healthy preschool children: $42 \%$ [28] and $43 \%$ [29].

\section{Conclusions}

In summary, our multicentre, multidevice study on normative respiratory impedance data for adult subjects revealed that FOT devices yield systematic differences in Rrs at 20 and $25 \mathrm{~Hz}$ and in $X \mathrm{rs}$ at frequencies $>14 \mathrm{~Hz}$. This indicates that the standardisation rules of the $Z_{\mathrm{rs}}$ measurements should be complemented with more rigorous calibration procedures in order to ensure data compatibility at all frequencies. However, for the lower-frequency $Z$ rs measures, the present prediction equations allow the comparison and interpretation of adult impedance data relating to Caucasians worldwide, and our data also provide information on the baseline variability of $\mathrm{Zr}$ and the bronchodilator response in healthy adults. The use of our baseline $Z$ rs data and the bronchodilator response should be evaluated prospectively to establish their ability to aid in the diagnosis and management of adult patients with asthma or chronic obstructive pulmonary disease.

\section{References}

Oostveen E, MacLeod D, Lorino $\mathrm{H}$, et al. The forced oscillation technique in clinical practice: methodology, recommendations and future developments. Eur Respir J 2003; 22: 1026-1041.

2 Beydon N, Davis SD, Lombardi E, et al. An official American Thoracic Society/European Respiratory Society statement: pulmonary function testing in preschool children. Am J Respir Crit Care Med 2007; 15: 1304-1345.

3 Làndsér FJ, Clément J, Van de Woestijne KP. Normal values of total respiratory resistance and reactance determined by forced oscillations: influence of smoking. Chest 1982; 81: 586-591.

4 Guo YF, Herrmann F, Michel JP, et al. Normal values for respiratory resistance using forced oscillation in subjects $>65$ years old. Eur Respir J 2005; 26: 602-608.

5 Brown NJ, Xuan W, Salome CM, et al. Reference equations for respiratory system resistance and reactance in adults. Respir Physiol Neurobiol 2010; 172: 162-168.

6 Pasker HG, Mertens I, Clément J, et al. Normal values of total respiratory input resistance and reactance for adult men and women. Eur Respir Rev 1994; 4: 134-137.

7 Clément J, Làndsér FJ, van de Woestijne KP. Total resistance and reactance in patients with respiratory complaints with and without airways obstruction. Chest 1983; 83: 215-220.

8 Pasker HG, Schepers R, Clément J, et al. Total respiratory impedance measured by means of the forced oscillation technique in subjects with and without respiratory complaints. Eur Respir J 1996; 9: 131-139.

9 James AL, Knuiman MW, Divitini ML, et al. Associations between white blood cell count, lung function, respiratory illness and mortality: the Busselton Health Study. Eur Respir J 1999; 13: 1115-1119.

10 American Thoracic Society. Standardization of spirometry, 1994 update. Am J Respir Crit Care Med 1995; 152 : $1107-1136$.

11 Quanjer PH, Tammeling GJ, Cotes JE, et al. Lung volumes and forced ventilatory flows. Report Working Party Standardization of Lung Function Tests, European Community for Steel and Coal. Official Statement of the European Respiratory Society. Eur Respir J 1993; 6: Suppl. 16, 5-40.

12 Brown H, Prescott R. Applied Mixed Models in Medicine. Chichester, John Wiley \& Sons, 2001.

13 Quanjer PH, Stanojevic S, Cole TJ, et al. Multi-ethnic reference values for spirometry for the 3-95-yr age range: the global lung function 2012 equations. Eur Respir J 2012; 40: 1324-1343.

14 Goldman MD. Clinical application of forced oscillation. Pulm Pharmacol Ther 2001; 14: 341-350.

15 Bland JM, Altman DG. Statistical methods for assessing agreement between two methods of clinical measurement, Lancet 1986; 1: 307-310.

16 World Health Organization. Obesity and Overweight Fact Sheet. www.who.int/mediacentre/factsheets/fs311/en Date last accessed: July 2009. Date last updated March 2013.

17 Zerah F, Harf A, Perlemuter L, et al. Effects of obesity on respiratory resistance. Chest 1993; 103: 1470-1476.

18 Yap JCH, Watson RA, Gilbey S, et al. Effects of posture on respiratory mechanics in obesity. J Appl Physiol 1995; 79: 1199-1205. 
19 Gimeno F, van der Weele LT, Koëter GH, et al. Forced oscillation technique. Reference values for total respiratory resistance obtained with the Siemens Siregnost FD5. Ann Allergy 1992; 68: 155-158.

20 Govaerts E, Cauberghs M, Demedts M, et al. Head generator versus conventional technique in respiratory input impedance measurements. Eur Respir Rev 1994; 4: 143-149.

21 Zerah F, Lorino AM, Lorino $\mathrm{H}$, et al. Forced oscillation technique vs spirometry to assess bronchodilatation in patients with asthma and COPD. Chest 1995; 108: 41-47.

22 Skloot G, Goldman M, Fischler D, et al. Respiratory symptoms and physiologic assessment of ironworkers at the World Trade Center disaster site. Chest 2004; 125: 1248-1255.

23 Nair A, Ward J, Lipworth BJ. Comparison of the bronchodilator response in patients with asthma and healthy subjects using spirometry and oscillometry. Ann Allergy Asthma Immunol 2011; 107: 317-322.

24 Peslin R, Jardin P, Duvivier C, et al. In-phase rejection requirements for measuring respiratory input impedance. J Appl Physiol 1984; 56: 804-809.

25 Van Noord JA, Clément J, Van de Woestijne KP, et al. Total respiratory resistance and reactance in patients with asthma, chronic bronchitis, and emphysema. Am Rev Respir Dis 1991; 143: 922-927.

26 Pham QT, Bourgkard E, Chau N, et al. Forced oscillation technique (FOT): a new tool for epidemiology of occupational lung diseases? Eur Respir J 1995; 8: 1307-1313.

27 Pairon JC, Iwatsubo Y, Hubert C, et al. Measurement of bronchial responsiveness by forced oscillation technique in occupational epidemiology. Eur Respir J 1994; 7: 484-489.

28 Thamrin C, Gangell CL, Udomittipong K, et al. Assessment of bronchodilator responsiveness in preschool children using forced oscillations. Thorax 2007; 62: 814-819.

29 Oostveen E, Dom S, Desager K, et al. Lung function and bronchodilator response in 4-year-old children with different wheezing phenotypes. Eur Respir J 2010; 35: 865-872. 